\title{
First results from the ESO VLTI calibrators program ${ }^{\star}$
}

\author{
A. Richichi and I. Percheron
}

\begin{abstract}
European Southern Observatory, Karl-Schwarzschildstr. 2, 85748 Garching bei München, Germany e-mail: arichich@eso.org
\end{abstract}

Received 26 October 2004 / Accepted 22 December 2004

\begin{abstract}
The ESO Very Large Telescope Interferometer (VLTI) is one of the leading interferometric facilities. It is equipped with several 8.2 and $1.8 \mathrm{~m}$ telescopes, a large number of baselines up to $200 \mathrm{~m}$, and with several subsystems designed to enable high quality measurements and to improve significantly the limits of sensitivities currently available to long-baseline interferometry. The full scientific potential of the VLTI can be exploited only if a consistent set of good quality calibrators is available. For this, a large number of observations of potential calibrators have been obtained during the commissioning phase of the VLTI. These data are publicly available. We briefly describe the interferometer, the VINCI instrument used for the observations, the data flow from acquisition to processed results, and we present and comment on the volume of observations gathered and scrutinized. The result is a list of 191 calibrator candidates, for which a total of 12066 observations can be deemed of satisfactory quality. We present a general statistical analysis of this sample, using as a starting point the angular diameters previously available in the literature. We derive the general characteristics of the VLTI transfer function, and its trend with time in the period 2001 through mid-2004. A second paper will be devoted to a detailed investigation of a selected sample, aimed at establishing a VLTI-based homogeneous system of calibrators.
\end{abstract}

Key words. techniques: high angular resolution - techniques: interferometric - catalogs - stars: fundamental parameters

\section{Introduction}

Long-baseline interferometers provide at present the highest angular resolution possible from the ground at optical and infrared wavelengths. In the last few years, the CHARA, Keck and VLTI interferometers (ten Brummelaar et al. 2003; Colavita \& Wizinowich 2003; Glindemann et al. 2003) have started operation, while other facilities such as OHANA (Perrin et al. 2004) and MRO (Creech-Eakman et al. 2003) are being readied. These new large interferometers couple for the first time hectometric baselines with large telescopes, offering a wavelength coverage from the visual to the thermal infrared and aiming also at nulling interferometry.

Ground-based long-baseline interferometry (LBI) is a technique that is heavily affected by atmospheric turbulence. This sets limits on the integration times, on the quality of the wavefronts that are combined from the various telescopes, and in general on the accuracy of the measurements. Although significant improvements can be obtained by selecting observational sites of excellent quality and by adopting techniques such as adaptive optics, fringe tracking and beam filtering through monomode fibers, the process of calibration remains a fundamental requisite for the success of interferometric measurements, and this aspect has only become more pressing in view

* Table 4 is only available in electronic form at the CDS via anonymous ftp to cdsarc.u-strasbg.fr $(130.79 .128 .5)$ or via http://cdsweb.u-strasbg.fr/cgi-bin/qcat? J/A+A/434/1201 of the needs in terms of accuracy demanded by the most recent interferometers mentioned above. Indeed, the accuracy of calibration is at present the limiting factor in measurements performed with the VLTI, which additionally is located in the southern hemisphere where interferometric calibrators have been traditionally more scarce.

The problem of calibration, although crucial for the success of interferometric observations, has remained until now a relatively uncoordinated effort. In general, the tendency has been to use different, often independent lists of calibrators at each interferometric facility. Another approach has been to estimate the angular diameter for a set of suitable stars. Cohen et al. (1999) have produced a list of over 400 sources, with typical magnitudes and diameters which are well suited mostly for baselines of less than $100 \mathrm{~m}$ and relatively small telescopes. Bordé et al. (2002) have refined this list, imposing selection criteria derived from their experience at the IOTA interferometer, while Mérand et al. (2005) have extended it to stars with smaller angular diameter and suitable for hectometric baselines.

The recent CHARM2 catalogue (Richichi et al. 2005) is an effort to include all known direct measurements at high angular resolution of angular diameters and binary stars, as well as indirect estimates such as those mentioned above. However, the best approach to the problem of calibrators, at least those that can be resolved by interferometric facilities, is undoubtedly represented by direct observations. 
In this paper, we present the first results of an effort to establish a network of calibrators for the VLTI. We review the main characteristics of the interferometer and its instrumentation, and the observations carried out as part of the VLTI commissioning. We also briefly describe the VINCI instrument used for such observations, and the process of automated data reduction employed. We then present the list of observed objects and the main statistical conclusions, including an assessment of the performance of the VLTI, its accuracy and stability. A detailed analysis and discussion of individual results is the subject of a second paper.

\section{VLTI overview}

The ESO Very Large Telescope Interferometer (VLTI), located on Cerro Paranal in Chile, is based on the same site and infrastructure as the widely known VLT observatory (Glindemann et al. 2000). In addition to the four $8.2 \mathrm{~m}$ unit telescopes (UT), it includes a number of $1.8 \mathrm{~m}$ auxiliary telescopes (AT). These are expecially designed for interferometry, with very compact structures and an optical system which replicates the light path inside the UT telescopes (Koehler \& Flebus 2000). Currently, one AT is available, two more are being delivered and a fourth one is in an advanced phase of construction. Also, two $0.4 \mathrm{~m}$ siderostat test telescopes (SID, Derie et al. 2000) are available, which have represented the workhorse of the commissioning activities of the VLTI (Schöller et al. 2003). The ATs and SIDs can be moved over an array of 30 stations. In particular, the ATs can be moved semi-automatically on a system of tracks and can be relocated in a matter of hours; this is a unique feature among large optical interferometers. Altogether, 254 independent baselines are available, leading to 3025 closure phases, with a rather homogenous distribution in baseline lengths (from 8 to $205 \mathrm{~m}$ ) and orientations (Richichi et al. 2000).

The VLTI is following a dense implementation plan, with several subsystems and instruments becoming available and being commissioned in close sequence (Schöller et al. 2003). Table 1 provides an overview of the main steps. More details can be found in Derie (2000) for the delay lines, Gai et al. (2003) for FINITO, Donaldson et al. (2000) for MACAO, Koehler \& Flebus (2000) for the ATs, Derie et al. (2003) for PRIMA.

The VLTI instruments and their characteristics are crucial in defining the science capabilities of the interferometer, and the specifications on the calibrators. The first of these instruments is VINCI, developed by the Paris Observatory in collaboration with ESO (Kervella et al. 2000). It achieved first fringes with the SIDs in March 2001, and with the UTs in October 2001. VINCI operates with a broad $K$-band filter using a Hawaii array as detector. For most of its operation, VINCI has been used with the fiber-based beam combiner MONA (see Sect. 3). Note that at the time of writing another beam combiner is being used with VINCI, based on integrated optics (IONIC, Laurent et al. 2002; Le Bouquin et al. 2004). IONIC is available in two versions, that cover the $H$ and the $K$ bands respectively. No extensive calibrator observations have been collected and made public yet with IONIC, and we will restrict ourselves in
Table 1. Schedule of VLTI subsystems and instruments.

\begin{tabular}{lll}
\hline \hline System & \multicolumn{1}{c}{ Description } & Status (Oct. 04) \\
\hline VINCI & Test instrument & available \\
MIDI & $10 \mu$ m instrument & available \\
DL 1-6 & Six Delay Lines & available \\
AMBER & 1-2.4 $\mu$ m instrument & commissioning \\
FINITO & IR Fringe tracker & commissioning \\
MACAO 1-3 & UT Adaptive Optics & available \\
MACAO 4 & UT Adaptive Optics & integration \\
AT 1 & Auxiliary telescope & available \\
AT 2 & Auxiliary telescope & integration \\
AT 3\&4 & Auxiliary telescopes & Europe \\
SID & Test Siderostats & to be decomm. \\
PRIMA & Dual-feed \& phase & from 2006 \\
& \multicolumn{2}{c}{ referencing } \\
\hline
\end{tabular}

Table 2. Summary of VLTI first generation instruments and their characteristics related to the needs of calibration.

\begin{tabular}{lccc}
\hline \hline System & VINCI & MIDI & AMBER \\
\hline Bands & $K$ & $N$ & $J, H, K$ \\
$\lambda \lambda(\mu \mathrm{m})$ & $1.9-2.4$ & $8-13$ & $1.0-2.4$ \\
Ang. resol. $^{a}(8 \mathrm{~m})$ & 38.7 & $182-364$ & $22.7-40.0$ \\
Ang. resol. $^{a}(202 \mathrm{~m})$ & 1.5 & $7.2-14.4$ & $0.9-1.6$ \\
Spectr. resol. $(\lambda / \Delta \lambda)_{\text {Beams }}^{5}$ & $30-260$ & $35-14000$ \\
Diff. phase & 2 & 2 & 3 \\
Polarization & no & no & yes \\
Accuracy $(\Delta V / V)$ & no & no & no \\
Lim. magnitude & $10^{-3}$ & $10^{-2}$ & $10^{-4}$ \\
\hline
\end{tabular}

${ }^{a}$ Expressed in 0.001 for $50 \%$ visibility at the central wavelengths of the shortest and longest band, when applicable.

${ }^{b}$ Effectively achieved during commissioning, in $K$-band, using MACAO.

${ }^{c} \mathrm{~N}$-band magnitude (goal) for operation with the FINITO fringe tracker. From Leinert et al. (2003).

${ }^{d} K$-band magnitude (goal) for $5 \sigma$ fringe detection in $100 \mathrm{~ms}$ on UTs equipped with MACAO (Strehl =0.5). From Petrov et al. (2001).

this paper to VINCI operation in a broad-band $K$ filter with the MONA beam-combiner.

While VINCI was designed and built mainly to test and commission the VLTI, the two facility instruments are MIDI and AMBER, both of which are installed and operating at the VLTI. In particular, MIDI (Leinert et al. 2000) operates in the $N$ band, with a foreseen extension to the $Q$ band. It offers various levels of spectral resolution and it represents one of very few instruments of its kind in the world, and the only one in the southern hemisphere. AMBER (Petrov et al. 2000) is based on a concept of a fiber-based beam combination similar to VINCI. It offers 3 bands in the near-infrared with various levels of spectral resolution, and the possibility to combine either 2 or 3 beams. In order to achieve the highest possible accuracy on differential phase measurements demanded by some of its scientific goals, AMBER employs also a scheme of rapid beam switching.

The main characteristic of the VLTI instruments are summarized in Table 2. We stress that in the case of the AMBER and MIDI instruments, which are still in a preliminary phase of 


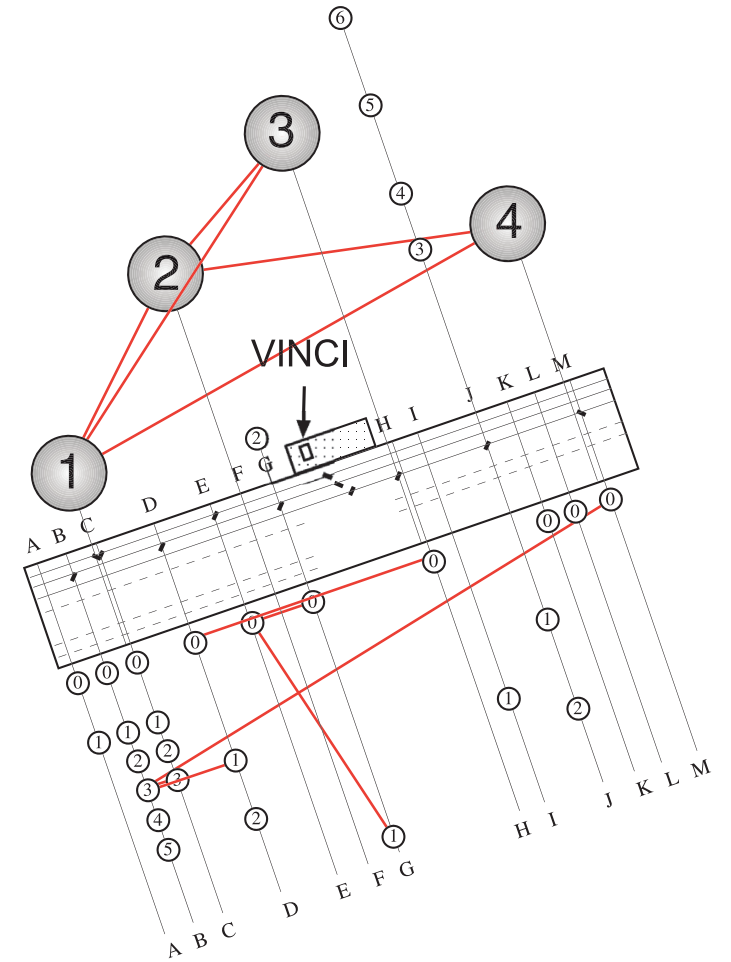

Fig. 1. Schematic layout of the VLTI interferometer, showing the baselines used for the calibrators observations as listed in Table 3. North is up.

operation without the full VLTI or not entirely within specifications, the values listed are relative to the foreseen performance only. Of particular interest are the angular resolution, the sensitivity limits and the accuracy of the visibility measurements. These represent the main parameters required for the selection of VLTI calibrators. However, a number of additional steps are currently under study, that could change significantly the scenario provided so far. For example, the dual-feed PRIMA facility (Derie et al. 2003) will push by several magnitudes the sensitivity limits, in those cases in which a nearby bright star could be used for fringe tracking. It is foreseen that the limiting sensitivity in $\mathrm{K}$ band would then reach about 20 mag about $4 \mathrm{~h}$ of integration. ESO and the European Space Agency ESA are currently studying the possibility to build GENIE, a ground demonstrator of the DARWIN nulling interferometer that will operate later in space (Fridlund et al. 2003). Finally, a number of concepts for a second generation of VLTI instruments are also being considered (Glindemann et al. 2003). For the immediate purpose of this paper, we will not discuss the implications of such developments, that would require a new set of calibrators.

\section{VINCI/MONA observations and data analysis}

The general layout of the VLTI system is shown in Fig. 1. Two afocal beams of light from either two siderostats or two UTs are reduced in diameter and sent through an underground light duct system to the interferometric tunnel where they are compensated for optical path differences by delay lines. The beams are then directed into the interferometric laboratory. Here, they are compressed in diameter from 80 to $18 \mathrm{~mm}$ (note this will not be required for AT operation), and are sent to the VINCI instrument where they are focused onto single-mode optical fibers and afterwords combined by a fiber beam combiner providing two interferometric outputs. Before beam combination, the photometric signals are separated from each of the two input beams in order to monitor the photometric fluctuations. This design allows the higher order modes of the incoming radiation to be filtered and achieves interference with beams which are only affected by the lower order modes, specifically by tiptilt induced by atmospheric piston noise. This permits optimal performance in terms of accuracy of visibility measurements. Thanks also to the stability of the VLTI environment, VINCI attains often uncalibrated visibility accuracies at the $0.1 \%$ level for many hours during nights of good seeing. This potential accuracy is often not fully exploited, due to the lack of suitable calibrators.

The two interferometric and two photometric output signals are imaged simultaneously on the detector. The fringes are detected by modulation of the optical path difference with an amplitude of typically $200 \mu \mathrm{m}$ to $300 \mu \mathrm{m}$ using a piezo element within the VINCI instrument, and kept within the scan length by sending an offset signal to the delay lines at slow rates of about $1 \mathrm{~Hz}$. The two interferometric outputs, which are in opposite phase, are subtracted to get a single interferogram which is free of systematic system noise. The squared coherence factor is obtained for each interferogram. A detailed description of the algorithms used in processing the VINCI data is given by Kervella et al. (2004). Note that two main approaches can be selected, based on Fourier and wavelet transforms respectively. Experience in the VLTI group with repeated measurements on the same source and with large volumes of data has shown that the two approaches lead essentially to the same results and accuracy, although the wavelet analysis is more accurate on single measurements. We have used this latter for our computations of the transfer function presented in Sect. 5. An observation consists of the execution of an observing block (OB), which is a sequence of typically 300 to 500 scans. This sequence is also called a batch.

A description of the VLTI data flow, in connection also with VINCI, can be found in Ballester et al. (2002, 2004). In summary, two data reduction systems are available. Firstly, an automated data reduction through the so-called pipeline is triggered by the execution of an OB on Paranal. A similar pipeline can be initiated manually off-line with parameter options in Garching as we did. This produces a synthetic ASCII summary called the quality control (QC) $\log$. QC logs include among others the source name, time of observation, baseline, telescope type, number of scans utilized, the coherence factor and errors of each interferogram as well as their average, and quality flags.

Secondly, various data reduction packages are available, some of which are based on the same core of algorithms as the pipeline with an added library of graphical interfaces and scan selection routines, and some of which start from the data produced by the pipeline and provide additional selection and fitting algorithms. This interactive data reduction is used for an accurate analysis of individual observations. However, for the 
Table 3. Statistics of the VINCI/MONA observations.

\begin{tabular}{lrrrr}
\hline \hline Baseline & $\begin{array}{c}\text { Length } \\
(\mathrm{m})\end{array}$ & Nights & $\begin{array}{r}\text { Total } \\
\text { \# OB }\end{array}$ & $\begin{array}{r}\text { Accepted } \\
\text { Cal. \# OB }\end{array}$ \\
\hline B3-C3 & 8.0 & 27 & 860 & 426 \\
B3-D1 & 24.0 & 63 & 2675 & 1667 \\
B3-M0 & 139.7 & 81 & 1856 & 1118 \\
D0-H0 & 64.0 & 50 & 825 & 690 \\
E0-G0 & 16.0 & 294 & 8317 & 5606 \\
E0-G1 & 66.0 & 145 & 3147 & 2188 \\
U1-U2 & 56.6 & 1 & 8 & 11 \\
U1-U3 & 102.4 & 15 & 422 & 250 \\
U1-U4 & 130.2 & 1 & 41 & 9 \\
U2-U3 & 46.6 & 9 & 149 & 85 \\
U2-U4 & 89.4 & 2 & 52 & 16 \\
\hline Total & & 688 & 18352 & 12066 \\
\hline
\end{tabular}

large volume of calibrator observations discussed here this approach is not practical. We reprocessed all the raw data relevant to this paper with a single homogeneous version of the pipeline installed at the ESO headquarters in Garching, and we used the average values present in the QC logs.

Note that the pipeline applies flags to filter out individual scans and full OBs which do not meet a number of criteria. The settings that we used were such that a scan was rejected if the signal-to-noise ratio (SNR) on the two photometric beams differed by more than a factor of 5 ; if the fringe packet was too wide or too narrow in the time domain (more than $50 \%$ away from the theoretical value); if the fringe peak was not at the correct position in the frequency domain (more than $30 \%$ difference from the theoretical position); if the fringe peak was too wide or too narrow in the frequency domain (more than $40 \%$ difference from the theoretical value); if there was an optical path difference (OPD) jump of more than $20 \mu \mathrm{m}$ before the scan; if fringes were located at edge of the scan; and if the scan visibility was more than $3 \sigma$ from the OB average. A full $\mathrm{OB}$ was rejected if less than 10 interferograms could be successfully processed; if less than $5 \%$ of the scans could be successfully processed; if the photometric signal averaged SNR less than 1. More details on these criteria can be found in the paper by Kervella et al. (2004).

A summary of the total observations, ordered by baseline, is given in Table 3 . The last two columns list for each baseline, respectively, the total number of OBs succesfully processed and the number of candidate calibrator OBs which were accepted after the tests mentioned above. Our definition of a candidate calibrator is provided in Sect. 4 , and more details on the data statistics are given in Sect. 5.

\section{The interferometric transfer function}

The squared visibility of a source with angular diameter $\phi$, observed with an interferometer having a baseline $B$, at the wavelength $\lambda$, is defined as

$$
V^{2}=\left[\frac{2 J_{1}(\pi \phi B / \lambda)}{\pi \phi B / \lambda}\right]^{2} \text {. }
$$

Here, and throughout the paper, we assume always a uniform stellar disc. The effect of limb-darkening can be investigated by long-baseline interferometry (Wittkowski et al. 2001, 2004), but it is relatively small and can be neglected for the purpose of this paper. Due to degradations introduced by the atmosphere, as well as by the optics and mechanics of the interferometer, the actual squared visibility observed is $V_{\mathrm{o}}^{2}$, with $V_{\mathrm{o}}^{2}<V^{2}$. The transfer function $\mathrm{TF}$ is defined as the ratio

$\mathrm{TF}=V_{\mathrm{o}}^{2} / V^{2}$

In order to perform scientific measurements on stars with an unknown angular diameter, it is necessary to estimate TF from stars for which the angular diameter is known. This is in essence the process of calibration of an interferometer. Since the TF is subject to the influence of atmospheric turbulence, it must be measured as frequently as possible. It is also desirable to estimate it from stars with a similar elevation, and ideally also in a similar direction to the scientific target stars.

It can be assumed that the process of determining an interferometric transfer function and the guidelines for its validity could be similar to those established for example in studies of speckle interferometry (Aitken 1989; Leinert 1994). We note however that some differences could exist, for example linked to the different atmospheric properties over distances significantly longer than the outer turbulence scale, and this effect could be site-dependent (Abahamid et al. 2004). There is no general study of the regime of validity of transfer function determinations for long-baseline interferometry.

For the purpose of this paper, we have used the whole database of VINCI measurements after automated processing by the pipeline, using only OBs which were not flagged for rejection as explained in Sect. 3. We have identified a number of stars which satisfied the main criteria to be considered as candidate calibrators. These include the stability, the absence of nearby companions, and suitable spectral types. More details are given by Percheron et al. (2003). A summary of the observations satisfying these criteria is given in the last column of Table 3 .

Essentially, we have used as the main source the VLTI Calibrator catalog available to users of the ESO web tools. This in turn is based on entries from CHARM2 (Richichi et al. 2005), from the list of MIDI calibrators (T. Verhoelst, private communication) and from the catalog of Bordé et al. (2002). Very few stars come from other miscellaneous sources. Details are given in the on-line material (see Table 4). Our list includes stars which cannot always be considered good calibrators in a strict sense. In particular, since MIDI is an instrument working in the $10 \mu \mathrm{m}$ range, the compilers of its list of calibrators were less inclined to reject stars with some known variability in the near-IR, as long as it did not affect its mid-IR behaviour. As a result, some of the MIDI calibrators have quite large angular diameters and/or are variable in the near-IR. We do not concern ourselves here with this issue, and we postpone a critical selection to the next paper.

On the basis of the available angular diameters, we have computed the $\mathrm{TF}_{i, j}$ for each calibrator $j$ and for each night $i$, 
and the associated error $\Delta \mathrm{TF}_{i, j}$. We have then computed for each night the weighted average transfer function and its error

$$
\begin{gathered}
\mathrm{TF}_{i}=\frac{\sum_{j} \Delta \mathrm{TF}_{i, j}^{-2} \cdot \mathrm{TF}_{i, j}}{\sum_{j} \Delta \mathrm{TF}_{i, j}^{-2}} \\
\Delta \mathrm{TF}_{i}=\left(\sum_{j} \Delta \mathrm{TF}_{i, j}^{-2}\right)^{\frac{1}{2}} .
\end{gathered}
$$

We stress that this is a rather rough approximation, since it is based on a pre-defined set of $\phi_{j}$ values and we know that many of the listed calibrator diameters are not accurate to the precision demanded by the VLTI/VINCI measurements. The values we have used are those provided in the references mentioned above, and in many cases the diameters are either for a wavelength other than the $K$ band, or several determinations are available. We have not attempted to derive a definitive value for each star, but have taken instead what we considered a representative value. Nevertheless, we estimate that the derived TF as a function of time is a sufficient approximation for a statistical discussion as provided in Sect. 5. It will be the subject of a second paper to go into more detail for a selected sample of calibrators, and in particular to provide refined angular diameter values. Note that the error terms $\Delta \mathrm{TF}_{i, j}$ include also the uncertainties on the angular diameters $\Delta \phi_{j}$, which at this stage are necessarily as tentative as the initial values of angular diameters themselves.

\section{Statistics of the observations}

The VLTI started operations in the middle of March 2001, using the VINCI instrument and the SID. Since then, observations have continued almost uninterrupted on every useful night. The main goal of this long period of observations has been the commissioning of the VLTI, including the characterization of several baselines (see Fig. 1), of the delay lines, of the several subsystems, and of the complex software overviewing the whole system (Schöller et al. 2003). More recently, scientific observations have started, including the so-called Science Demonstration (SD), Guaranteed Time Observations (GTO) for MIDI, and Open Time (OT) proposals by the community. All commissioning and SD data taken on the sky with possible scientific relevance are being released to the public on a regular basis.

These public data form the basis of the material discussed in this work. We have considered observations obtained from the night of first fringes, 16 March 2001, to 22 July 2004, which corresponds to the latest public release. This makes a total of 1225 nights. The actual number of nights with data useful for our reprocessing is about $60 \%$ of this, as detailed in Table 3. The rest of the time was spent mostly on integration and commissioning activities with VINCI as well with other instruments, not on the sky, or for GTO and OT scientific observations which are protected by proprietary rules. Overall, the fraction of time in which the VLTI was idle from observations or technical work was less than 2 months over the first 3.4 years, which represents in itself an impressive performance.

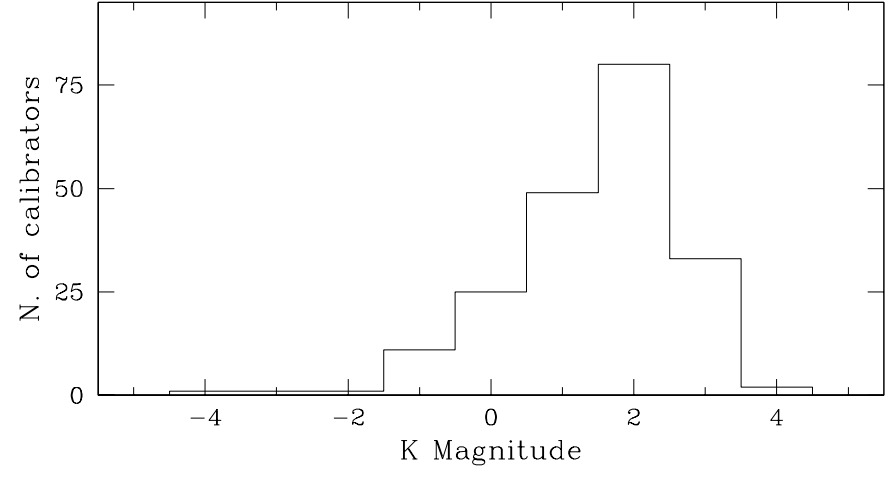

Fig. 2. $K$ magnitude distribution of the observed candidate calibrators.

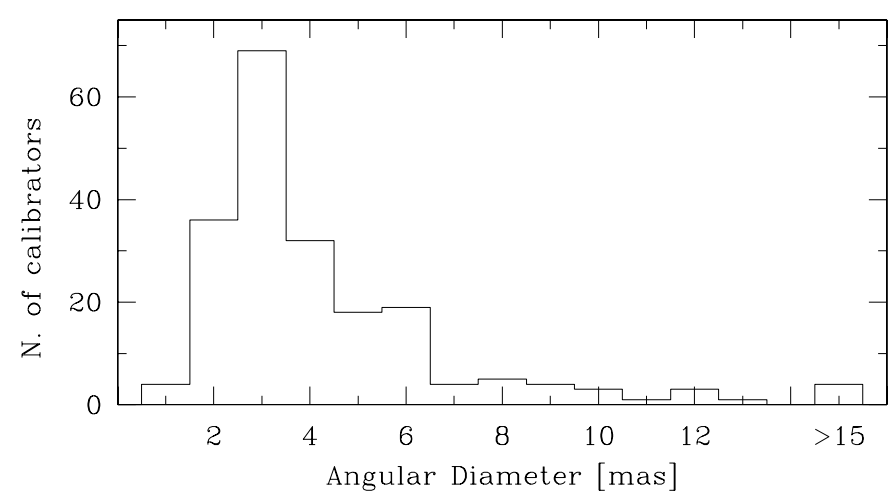

Fig. 3. Angular diameter distribution of the observed candidate calibrators.

Some general statistics are presented in Table 3. The total volume of data processed amounted to $20849 \mathrm{OBs}$, or $\approx 150 \mathrm{~Gb}$ of raw FITS files. Of these, 18402 were successfully processed by the pipeline. Note a slight difference to the total number provided in Table 3, because for 50 OBs the baseline information was not reliable. After the selections described in Sect. 3, 15123 OBs were accepted as having a satisfactory quality, for a total of 287 stars. A significant fraction of these stars were actually science targets, or otherwise considered unsuitable as calibrators. After removing these entries, our database includes 12066 OBs of good quality on 191 candidate calibrators. Details are given in Table 4 , which is provided as online material. In particular, we provide the following: a list of the candidate calibrators; their main parameters such as coordinates, magnitudes, spectral types; the baselines on which they were observed and how many times.

Figures 2 and 3 show the magnitude and brightness distributions of the observed candidate calibrators. The $K$ magnitudes have been obtained following the same criteria and using the same information as described in Richichi et al. (2005). Note that nominal values of the diameters are used, as stressed in Sect. 4. It can be seen that most of the sources are within the brightness limits of $K=0$ and $K=3 \mathrm{mag}$. This is a consequence of the typical brightness limit attained with VINCI on the small SID telescopes. The detector integration times used with VINCI during the commissioning observations 


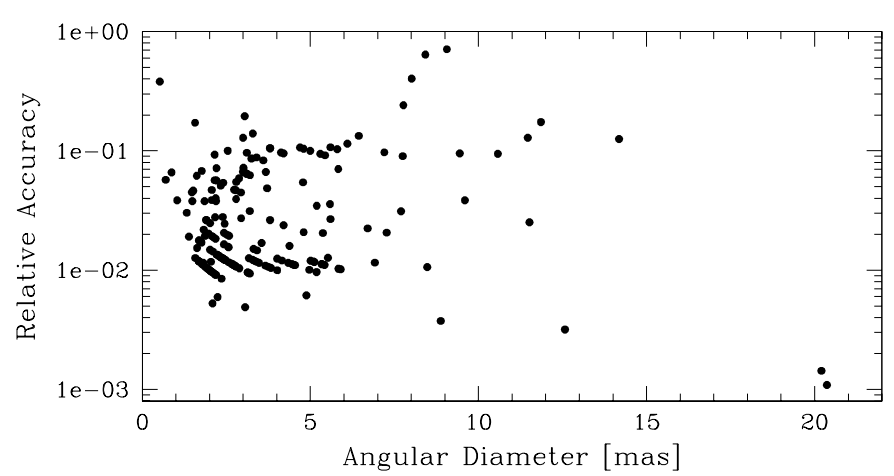

Fig. 4. Diameter accuracy distribution of the observed candidate calibrators.

varied between 0.1 and $20 \mathrm{~ms}$, however the most commonly used values (accounting for $80 \%$ of the data) were between 0.8 and $1.0 \mathrm{~ms}$. These should be understood as time spent on a scan. When the total number of scans in an OB is considered, the corresponding typical integration times were about 0.4 to $0.45 \mathrm{~s}$.

Also, it can be noted from Table 3 that the majority of the observations were carried out on SID telescopes, with the UTs accounting for only $3.0 \%$ of the total number of accepted OBs. We stress that for the present work only observations on UTs without MACAO were used. Typically, there is a difference of about $\lesssim 4-5$ mag between the performance of VINCI at the UTs without MACAO and at the SID. However, the VLTI Calibrator catalog was based on the SID performance and this explains why Fig. 2 does not extend to $K \approx 10$ which would be a more realistic limit for the UTs.

The angular diameters peak at 3 mas, providing a good coverage of near-IR calibrators especially for baselines up to about $100 \mathrm{~m}$. For longer baselines, this set of calibrators can be used but most of them will be significantly resolved and the accuracy of calibration could be affected. For longer wavelengths, such as in the $10 \mu \mathrm{m}$ range, all VLTI baselines can be adequately calibrated. It should be noted that a small number of candidate calibrators have very large angular diameters: these are mainly from the MIDI list, and are unsuitable for near-IR calibration.

Ideally, the best calibration accuracy is achieved when the calibrator candidate can be assumed to be effectively unresolved at the given wavelength and baseline. In practice, as just mentioned in the context of Fig. 3, calibrators can be marginally resolved. In this case, the relative accuracy with which the angular diameter is known will affect directly the accuracy of the final calibrated visibility of the science target. Figure 4 shows that about $70 \%$ of the observed candidate calibrators have angular diameters known, or presumed, with better than $5 \%$ accuracy. In this figure, it can be noted how some of the points with higher accuracy and smaller angular diameters seem to be lined up along a few precise curves. These are candidate calibrators for which the angular diameter has been determined indirectly, such as those present in the list of Cohen et al. (1999). The curves are the consequence of a precise link between the angular size and the accuracy of the determination, and are probably produced by the process of modelling and the associated parameter uncertainties.

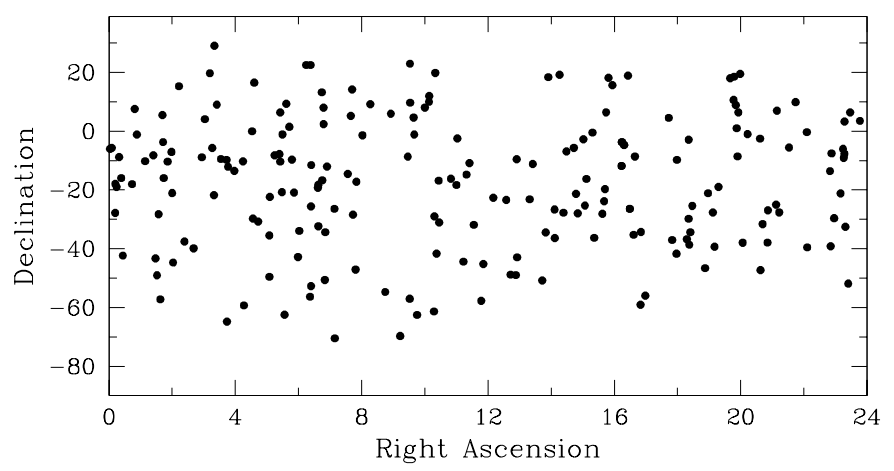

Fig. 5. Sky distribution of the observed candidate calibrators.

It can be appreciated from Fig. 5 how the sky distribution coverage is relatively uniform. For each of the 191 candidate calibrators, the nearest one is on average 5.8 , and never more than 15.8 , away. More important is the distance to the nearest calibrator for any point in the sky. Restricting the declination range between $-85^{\circ}$ and $+35^{\circ}$, equivalent to an airmass of 2 as seen from Paranal, the average distance to the nearest calibrator is 9.0 , and it never exceeds $30^{\circ}$. If the range of declinations is restricted so as to limit the airmass to no more than 1.5 , the average distance to the nearest calibrator is 7.0 , and it never exceeds $26^{\circ}$.

As mentioned in Sect. 4, unfortunately no precise studies are available on the dependence of the accuracy of calibration from the angular distance between science and calibrator sources. It is generally assumed that such a distance should be less than $\approx 15^{\circ}$ for reliable results, and it is probably desirable to keep it even smaller. Davis et al. (2004) found almost no changes in the VLTI transfer function over a wide range of zenith angle, although this study was admittedly limited to one night only. From this point of view, it appears that the coverage provided by the list of candidate calibrators observed by the VLTI is sufficient for most applications.

\section{The VLTI transfer function}

One main result that can be drawn from the large database of VLTI observations of candidate calibrators is the evaluation of the VLTI transfer function, its accuracy and its stability, both on a nightly basis and its long-term behaviour. For this, we have computed the transfer function TF according to the definitions given in Sect. 4. We stress again that our computations are based on a set of angular diameters, and their uncertainties, which at this stage are only first approximations. Given the large volume of observations and the number of candidate calibrators, we should not be affected by systematic biases in our conclusions. In order to further reduce this risk, we have chosen to use only nights in which at least 23 OBs were available. This number has been chosen as a compromise between having a good number of useful nights (about 200 according to this choice), and keeping at the same time only nights with a very high number of observations. A histogram of the number of nights as a function of OBs observed is shown in Fig. 6.

Additionally, we have chosen to discard from each night those OBs which produced a TF value departing by more than 


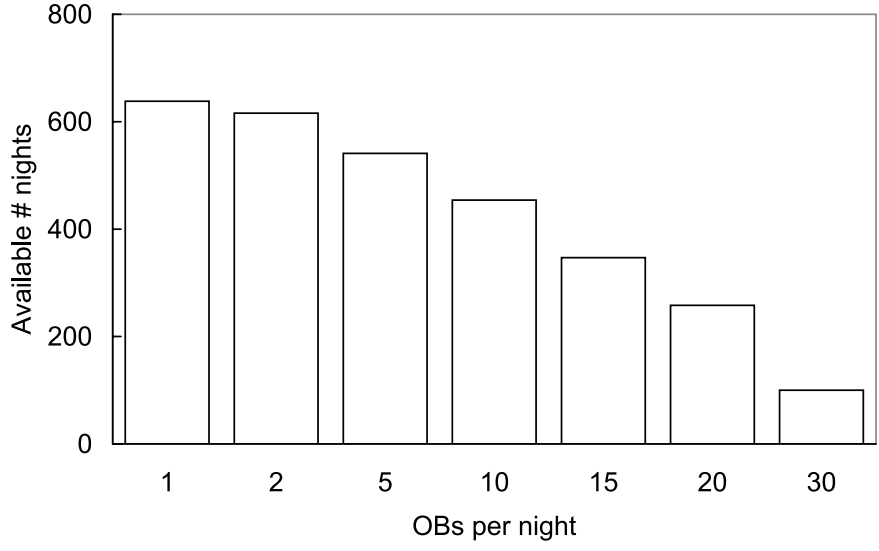

Fig. 6. Histogram of the number of nights according to the number of OBs (observation blocks) carried out on candidate calibrators. The maximum number of OBs ever observed in one night was 97.

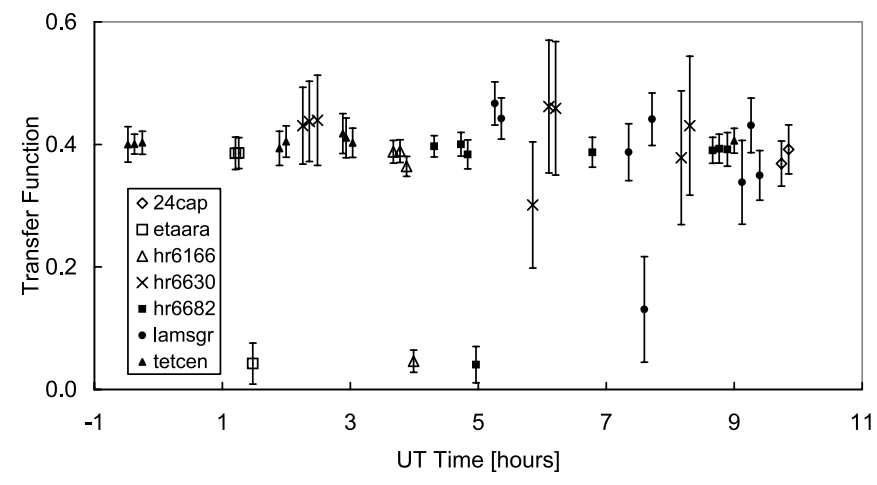

Fig. 7. Calibrators observations on the night of 2 June 2002, using the E0-G1 baseline. See the text for a discussion on the rejection of discrepant visibility points.

3 standard deviations $(\sigma)$ from the night average. Figure 7 shows an example in which three OBs recorded between 1 and $5 \mathrm{~h} \mathrm{UT}$ are obviously discrepant and were discarded according to the above criterium. It can be noted that another OB appears to be discrepant, around $7.5 \mathrm{~h} \mathrm{UT}$, but it was not discarded because it did not violate the $3 \sigma$ criterium.

On the other hand, in spite of this simple rejection criteria, the remaining validated $\mathrm{OBs}$ are not necessarily always satisfactory. An example is shown in Fig. 8, where intuitively one would be led to consider the OBs recorded after $6 \mathrm{~h}$ UT as discrepant. However, they do not differ by more than $3 \sigma$ from the night average. Also, it is difficult to find an obvious reason for this apparent discrepancy, since they are related to a $\operatorname{star}(\eta \mathrm{Sgr})$ for which several apparently good results were obtained earlier in the night, and no obvious correlation is seen with ambient parameters such as seeing, wind, coherence time.

We did not push our analysis to identify specific reasons for each rejection, or for the inclusion of apparently discrepant points. As stated earlier, our goal is mainly to derive global statistical properties, and we will devote another paper to the individual evaluation of selected results and to a refined list of angular diameters of selected calibrators. In particular, as mentioned in Sect. 4, some of the MIDI calibrators are known to be unsuitable for the near-IR.

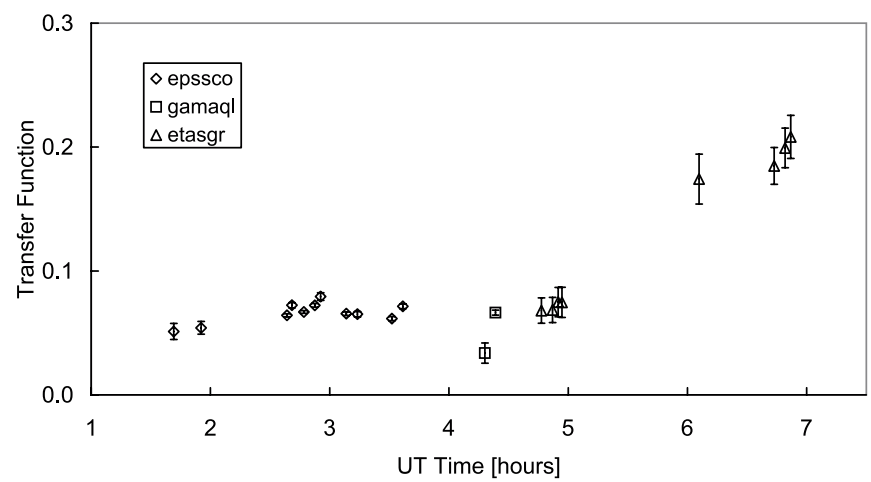

Fig. 8. Same as Fig. 7, for the night of 23 July 2001. The baseline used was E0-G0. No measurements were rejected, as explained in the text.

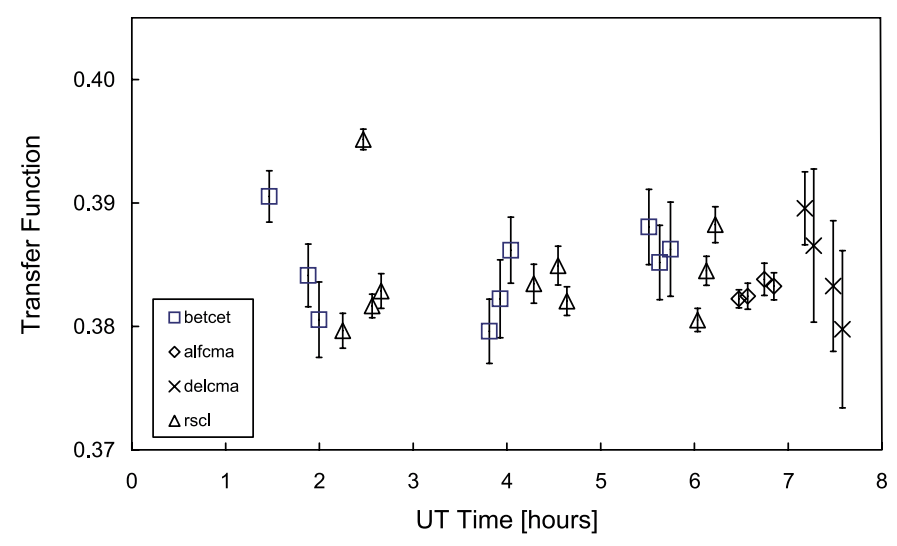

Fig. 9. Calibrators observations on the night of 14 November 2001, using the E0-G0 baseline. The weighted average transfer function is 0.384 with a relative error of only $0.5 \%$. This can be considered as an example of a good night at the VLTI.

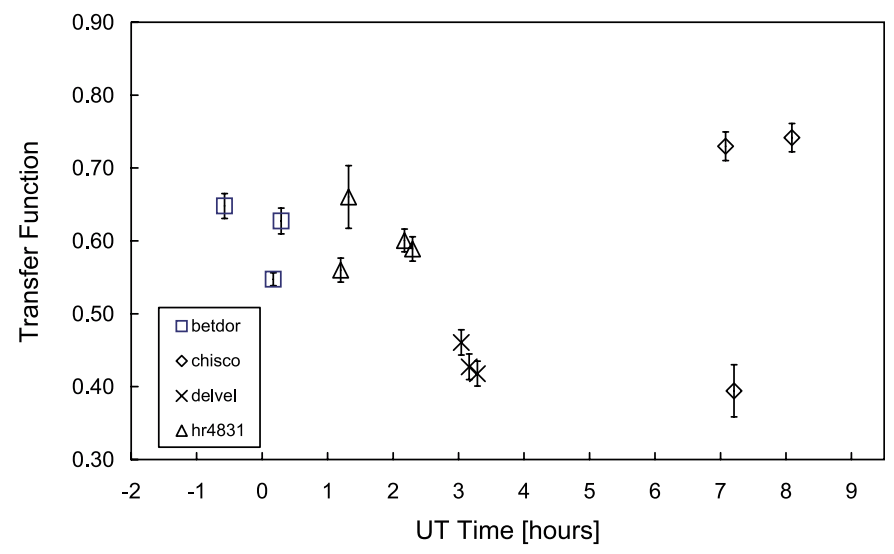

Fig. 10. Calibrators observations on the night of 20 April 2003, using the B3-M0 baseline. The weighted average transfer function is 0.574 with a large relative error of $15.8 \%$. This can be considered as an example of a very bad night at the VLTI. Note the large scatter in the measurements of each star.

Figures 9 and 10 show examples of two nights of calibrator observations, and the corresponding $\mathrm{TF}$ average value. It can be appreciated how, depending also on ambient conditions and in particular on the atmospheric seeing and coherence time, the observations might show different levels of scatter and quality. 


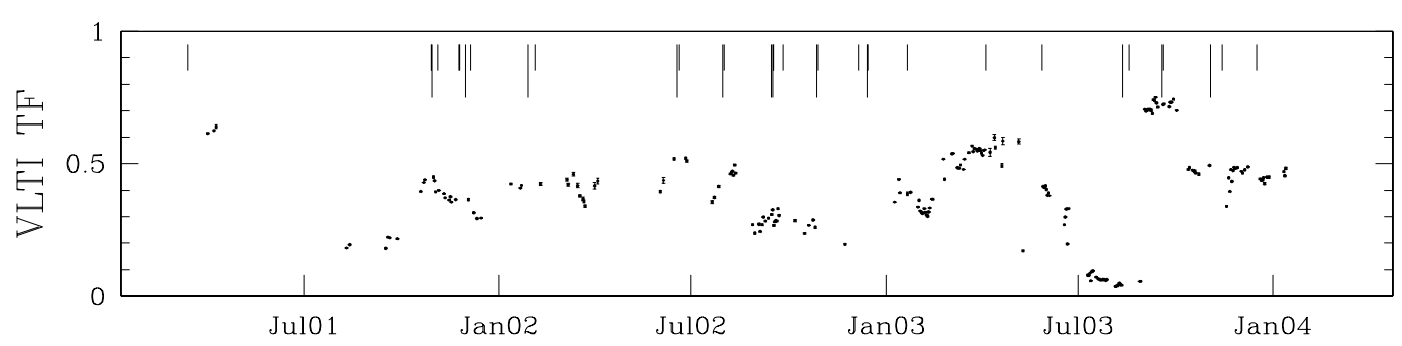

Fig. 11. Long term plot of the night-average VLTI transfer function, as computed from the nominal diameters of the candidate calibrators. Changes of baseline are marked by the vertical segments at the top (short for SID, long for UT baselines). At this scale, the error bars are barely visible.

On the night of November 14, 2001, the seeing was generally constant at the level of $0{ }^{\prime} 6$, increasing towards $11^{\prime \prime} 0$ only at the end of the night. The coherence time was also approximately constant around $4 \mathrm{~ms}$. Note that these values are computed at visual wavelengths, and at a location on the mountain top separate from the VLTI telescopes. On the night of April 20, 2003 on the contrary, the seeing fluctuations were more pronounced, with values between 0.3 and $1 ., 1$ and increasing to over 1 '.5 at the end of the night. Also the coherence time changed significantly during the night, ranging between 2 and $8 \mathrm{~ms}$.

The overall TF of the VLTI, computed under the assumptions illustrated so far, is shown in Fig. 11 for the totality of the available data. Firstly, one can notice a long period, in the summer of 2001, in which almost no transfer function is available. Indeed, in this period sky observations at the VLTI were almost completely halted, due to a problem in the MONA beam combiner. The problem, caused by temperature-dependent polarization of the single-mode fibers, recurred several times in the lifetime of this beam combiner. It required frequent adjustments of the local temperature of the fibers, and it became particularly worrysome in the summer of 2003, when a period of very low values of the transfer functions can be noticed. It should be stressed that this instability of the interferometric efficiency of the MONA beam combiner is characterized by relatively long time scales, of the order of weeks. As such, it is quite evident in the plot of Fig. 11, however it did not affect significantly observations collected during the same night.

A second consideration is that the VLTI transfer function varied at each baseline change, but not dramatically. This is due to the fact that such changes can involve movements and swaps of one or more relay mirrors in the delay line tunnel. In particular, it can be noted that there are no major systematic changes of the transfer function between SID and UT baselines. The biggest changes in the transfer function can be easily associated with temperature adjustments in the MONA beam combiner, realignments of the general optics, and similar interventions. When a baseline was in place for a long period of time, long-term drifts are evident, which can also be related to manual interventions on the components of the VLTI.

The smoothest part of the long-term transfer function is between October 19, 2001 and May 6, 2003. Before and after this range, the MONA problems mentioned above altered the long-term transfer function significantly. For the 115 nights inside this period, each having more than 23 OBs on calibrators, the average transfer function was $0.405 \pm 0.034$. Of course, it should be kept in mind that this relates to the long-term behaviour only. On a nightly basis the relative accuracies ranged from $0.1 \%$ to $2.9 \%$, with an average of $0.68 \%$ which can be considered as a satisfactory result.

\section{Conclusions}

We have presented and discussed a large set of interferometric observations of candidate calibrators obtained with the VLTI, equipped with the VINCI/MONA beam combiner, over a period of more than three years. The observations have been obtained in the framework of the VLTI commissioning, and are publicly available from the ESO web site. The data have been processed by an automated pipeline, and have been subjected to a number of quality criteria. A subset of 12066 observations has been selected.

From this, we have computed the VLTI transfer function and discussed its main statistical properties both on a nightly basis and in the long term. The typical transfer function accuracy (in the squared visibility sense) of the VLTI with VINCI/MONA of the order of $0.7 \%$ on a nightly basis, on a sample of 115 nights, each having at least 23 observations of candidate calibrators. For the long term behaviour, the transfer function shows marked fluctuations which generally can be explained in terms of slow degradations of the MONA beam combiner, and to a lesser extent in terms of baseline changes, realignments, and similar interventions. From October 2001 to May 2003, the average value of the transfer function was $0.405 \pm 0.034$

We present as on-line material a list of 191 candidate calibrators, together with their main characteristics and the statistics of their observations at the VLTI. This list is preliminary, in the sense that some of these sources, extracted from a catalog of calibrators for the mid-infrared beam combiner MIDI, are not ideally suited for calibration in the near-infrared. Also the angular diameter values are tentative, having been extracted from the available literature without any effort to perform a consistency check against the VLTI observations. Another paper is in preparation, with the goal of deriving a new set of angular diameters for a restricted sample of validated calibrators, by means of a global solution to the VLTI observations presented here.

Acknowledgements. The VLT Interferometer is the result of more than a decade of work in planning and engineering, and of several 
intense years of efforts on the site. We would like to recognize the many tens of people involved in this enterprise, even without being able to acknowledge each by name. This research has made use of the Simbad database, operated at the Centre de Données Astronomiques de Strasbourg (CDS), and of NASA's Astrophysics Data System Bibliographic Services (ADS).

\section{References}

Abahamid, A., Vernin, J., Benkhaldoun, Z., et al. 2004, A\&A, 422, 1123

Aitken, G. J. M. 1989, PASP, 101, 471

Ballester, P., Chavan, A. M., Glindemann, A., et al. 2002, SPIE Proc. 4844, ed. P. Quinn, 300

Ballester, P., Licha, T., McKay, D., et al. 2004, SPIE Proc. 5493, ed. J. M. Jr. Oschmann, 16

Bordé, P., Coudé du Foresto, V., Chagnon, G., \& Perrin, G. 2002, A\&A, 393, 183

Cohen, M., Walker, R. G., Carter, B., et al. 1999, AJ, 117, 1864

Colavita, M. M., \& Wizinowich, P. L. 2003, SPIE Proc. 4838, ed. W. A. Traub, 79

Creech-Eakman, M. J., Buscher, D., Haniff, C., et al. 2003, AAS, 202, 2403

Davis, J., Richichi, A., Ballester, P., et al. 2004, Astron. Nach., in press

Derie, F. 2000, Interferometry in Optical Astronomy, ed. P. Léna, \& A. Quirrenbach, SPIE Proc., 4006, 25

Derie, F., Brunetto, E., Duchateau, M., et al. 2000, Interferometry in Optical Astronomy, ed. P. Léna, \& A. Quirrenbach, SPIE Proc., 4006, 99

Hogenhuis, H., Visser, M., \& Derie, F. 2003, SPIE Proc. 4838, ed. W. A. Traub, 1148

Donaldson, R., Bonaccini, D., Brynnel, J., et al. 2000, Interferometry in Optical Astronomy, ed. P. L. Wizinowich, SPIE Proc., 4007, 82

Fridlund, M., \& Gondoin, P. 2003, Ap\&SS, 286, 93

Gai, M., Corcione, L., Lattanzi, M. G., et al. 2003, Mem. Soc. Astron. It., 74,472

Glindemann, A., Coudé du Foresto, V., Delplancke, F., et al. 2000, Interferometry in Optical Astronomy, ed. P. Léna, \& A. Quirrenbach, SPIE Proc., 4006, 2
Glindemann, A., Algomedo, J., Amestica, R., et al. 2003, SPIE Proc. 4838, ed. W. A. Traub, 89

Kervella, P., Coudé du Foresto, V., Glindemann, \& Hofmann, R. 2000, Interferometry in Optical Astronomy, ed. P. Léna, \& A. Quirrenbach, SPIE Proc., 4006, 31

Kervella, P., Ségransan, D., \& Coudé du Foresto, V. 2004, A\&A, 425, 1161

Koehler, B., \& Flebus, C. 2000, Interferometry in Optical Astronomy, ed. P. Léna, \& A. Quirrenbach, SPIE Proc., 4006, 13

Laurent, E., Rousselet-Perraut, K., Benech, P., et al. 2002, A\&A, 390, 1171

LeBouquin, J. B., Rousselet-Perraut, K., Kern, P., et al. 2004, A\&A, 424, 719

Leinert, C. 1994, in Star Formation and Techniques in Infrared and mm-Wave Astronomy, ed. T. P. Ray, \& S. V. W. Beckwith (Springer Verlag), 215

Leinert, C., Graser, U., Waters, L. B. F. M., et al. 2000, Interferometry in Optical Astronomy, ed. P. Léna, \& A. Quirrenbach, SPIE Proc., 4006, 43

Leinert, C., Graser, U., Richichi, A., Schöller, M., \& Waters, L. F. B. M. 2003, ESO Messenger, 112, 13

Mérand, A., Bordé, P., \& Coudé du Foresto, V. 2005, A\&A, 433, 1155

Percheron, I., Richichi, A., \& Wittkowski, M. 2003, Ap\&SS, 286, 105

Perrin, G., Lai, O., \& Woillez, J. 2004, AAS, 204, 816

Petrov, R., Malbet, F., Richichi, A., et al. 2000, Interferometry in Optical Astronomy, ed. P. Léna, \& A. Quirrenbach, SPIE Proc., 4006, 68

Petrov, R., Malbet, F., Richichi, A., et al. 2001, C. R. Acad. Sci. Paris, $\mathrm{t}, 67$

Richichi, A., Blöcker, T., Foy, R., et al. 2000, Interferometry in Optical Astronomy, ed. P. Léna, \& A. Quirrenbach, SPIE Proc., 4006, 80

Richichi, A., Percheron, I., \& Khristoforova, M. 2005, A\&A, 431, 773

Schöller, M., Gitton, P., Argomedo, J., et al. 2003, SPIE Proc. 4838, ed. W. A. Traub, 870

ten Brummelaar, T. A., McAlister, H. A., Ridgway, S. T., Turner, N. H., \& Sturmann, L. 2003, SPIE Proc. 4838, ed. W. A. Traub, 69

Wittkowski, M., Hummel, C. A., Johnston, K. J., et al. 2001, A\&A, 377, 981

Wittkowski, M., Aufdenberg, J. P., \& Kervella, P. 2004, A\&A, 413, 711 